\title{
Zeichen und die epistemische Asymmetrie
}

\section{Linguistic Signs and the Epistemic Asymmetry}

\section{Michal Rubáš}

\begin{abstract}
In communication, the mental and physical qualities get put into language. While the former can be ascribed to their bearers in the subject-use, the ascription of the latter is only possible in the object-use. The two ways of ascribing are very different, based on diverse epistemic capacities, and there is a unilateral dependency relation between them. The text raises the question whether these conditions imply some consequences for semiology.
\end{abstract}

\section{Keywords}

subject-use and object-use of predicates, language sign theory, P. F. Strawson, E. Husserl, M. Frank 


\section{Einleitung}

In der Kommunikation werden mentale sowie physische Eigenschaften versprachlicht. Erstere können ihren Trägern im sog. Subjekt-, letztere nur im Objektgebrauch zugeschrieben werden. Beiden Zuschreibungsweisen liegen grundverschiedene epistemische Vollzüge zugrunde und es herrscht zwischen ihnen eine einseitige Abhängigkeit. Der Text stellt die Frage, ob diese Verhältnisse besondere Anforderungen an semiologische Sprachtheorien implizieren.

Es werden zunächst im Folgenden die Motivationen und Analysen P. F. Strawsons und E. Tugendhats zum Thema sprachanalytischer Fundierung von (selbst)bewussten Erlebnissen wiedergegeben (1). Beide genannten Denker verbinden die Entdeckung der Inkonvergenz der epistemischen Positionen mit der Idee der die Kommunikation stabilisierenden semantischen bzw. grammatischen Symmetrie.

Dann wird Manfred Franks hermeneutische Kritik referiert, die die epistemische Asymmetrie ausnutzt als Beweis der Unhaltbarkeit jeder Annahme der semantischen Identität der Prädikate in der Kommunikation (2). In Strawsons und Franks sprachtheoretischer Auswertung ein und desselben erkenntnistheoretischen Phänomens stoßen wir also auf gegensätzliche Einschätzungen der Möglichkeit und Funktion des Bedeutungswandels der sprachlichen Ausdrücke in der Kommunikation. Das macht ihre Auseinandersetzung besonders aufschlussreich für die linguistische Semantik.

Zum Schluss werden aus diesem Debatte-Stand semiologische Konsequenzen gezogen, die letztendlich die phänomenologische Zeichenanalyse Edmund Husserls die Hauptrolle übernehmen lassen (3). Husserl stellen wir als den Denker vor, der Franks korrekte Kritik an Strawson konsequenter durchführen würde, weil er den Widersprüchen, denen Frank selbst dabei unterliegt, ausweicht.

\section{Bedeutungserhaltung trotz der epistemischen Asymmetrie (Strawson)}

Peter F. Strawson hat im Bereich der Sprachphilosophie eine schon klassische Analyse vorgelegt, deren Thema die Relation ist zwischen den semantischen (Sprach)Einheiten und deren epistemisch differenten Gegebenheitsweisen: in der Objekt- und der Subjektperspektive. Strawsons Untersuchung in Individuals wird durch die Leitfrage getragen, wie wir uns im Gespräch über ein Ding mit der Identität dieses Dinges vergewissern: Woran erkennen wir, praktisch formuliert, dass wir eben über dasselbe oder immer noch über dasselbe sprechen? In welchen impliziten Vollzügen realisiert sich die Präsentation, d. h. Identifikation und die Re-identifikation von einzelnen Gegenständen des Denkens (Strawson 1959, S. 15f)?

Unter einzelnen zu besprechenden Gegenständen herrsche Hierarchie und diese entspricht laut Strawson direkt den Weisen, wie wir uns auf einzelne Entitäten identifikationell beziehen. Manche Arten von Einzeldingen seien „fundamental“ (Strawson 1959, S. 11), andere in diesen fundiert und von ihnen abhängig. Auf die fundamentalen Indivi- 
duen wüssten wir uns direkt zu beziehen, auf die abhängigen nur mittels der (impliziten) Referenz auf die fundamentalen (Strawson, 1959, S. 17).

Für das entscheidende Identifikationsmittel hält Strawson die Ostension, und das gilt auch für die Fälle, wenn man indirekt, durch sog. Deskriptionen, referiert. Nach Strawson besteht keine Deskription „nur aus den allgemeinen Termen“, sondern sie sei immer eine Mischung von nicht-ostensiven und ostensiven Elementen. Deskription bedeute, dass man einen Teil des Universums, auf den nicht ostensiv referiert werden kann, in Beziehung zu demjenigen einführe, auf den ostensiv referiert wird. In einer Anspielung an Leibniz hebt Strawson hervor, dass das Einheitssystem der Zeit-Raum-Beziehungen, von dem erst Ostension ermöglicht werde, auch prinzipiell für eine einmalige Deskription notwendig sei und dass bloße „logisch individualisierende Beschreibung“ (Strawson 1959, S. 26), d. h. ein Definitionsdepot von allgemeinen Termen, nicht imstande ist, eine individualisierende Erkenntnis zu gebieten - es schließe weder eine leere noch eine mehrfache Anwendung (Referenz) aus.

Die Einmaligkeit der Referenz mittels der Deskription umfasst also notwendigerweise die Einführung des inkriminierten Objektes in einmalige Beziehung zu einem anderen, auf das man ostensiv referieren kann, d. h. das sich direkt in den Zeit-Raum-Rahmen verorten lässt. Die einmalige Beschreibung als Formulierung einer individualisierenden Tatsache müsse somit den Hinweis auf ein einmalig verortetes Seiendes im einheitlichen Zeit-Raum-Rahmen enthalten, was aber nicht bedeute, dass die einmalig deskribierte Entität selbst „in Raum und Zeit sein“ muss (Strawson 1959, S. 22).

So kann man nach Strawson mit der Antwort auf die Frage nach minimalen Bedingungen einer seitens Hörers gelungenen Identifikation des vom Sprecher thematisierten Einzeldings schließen: dieses Einzelding muss ein Mitglied unseres allgemeinen Erkenntnisrahmens der Einzeldinge sein (Strawson 1959, S. 24), der Hörer muss einen individualisierenden Fakt (individuating fact, Strawson 1959, S. 23) von diesem Einzelding kennen und wissen, dass jetzt darauf referiert wird (Strawson 1959, S. 23).

Der Hörer müsse imstande sein, anzuführen, wann und wo sich das betroffene Einzelding befindet oder befand. Es ist damit laut Strawson ebenfalls die Kenntnis seiner eigenen Position im Zeit-Raum-Rahmen notwendigerweise verknüpft: Wenn er seine eigene Verortung nicht kennt, sei er nicht imstande, anderes zu lokalisieren, die Ostension wäre unwirksam (Strawson 1959, S. 29).

Die Ostension scheint auf die jetzige Beobachtung beschränkt zu sein, und kein Beobachten ist ununterbrochen und unbegrenzt. Die Begriffsstruktur, die wir nach Strawson haben, setze aber zumindest einige dauerhafte Objekte mit numerischer Identität voraus (Strawson 1959, S. 34). Eine Humesche Reminiszenz hat nun Strawson im Sinne mit der Frage, wie in unserer Erfahrung zu differenzieren ist zwischen der numerischen und einer bloßen „qualitativen Identität“ (Strawson 1959, S. 34) der sich gebenden Entitäten. Bloße qualitative Gleichheit der Objekte würde keinen einheitlichen räumlichen Rahmen konstituieren, sondern dieser würde in viele unabhängige Systeme zerfallen

1 So kann ich einmalig auf ein neu gebautes Haus durch die begleitende Referenz auf ein bekanntes Gebäude in der Nachbarschaft referieren oder ich kann auf Johns Absicht, das Haus zu verkaufen, die nichts Räumliches ist, durch die Referenz auf John Bezug nehmen (meine Illustration). 
(Strawson 1959, S. 34). Die Ablehnung des skeptischen Atomismus Humes², die er gründlicher in seiner späteren Analysis and Metaphysics ausarbeitet, benutzt Strawson hier als Gelegenheit zur Hervorhebung der „komplexen Verbindung“ der Begriffe von Ort und Ding und zur Feststellung, dass wir neben den Dingen komplementär imstande sein müssen, auch „Stellen“ (places) im Zeit-Raum-Netz zu identifizieren (Strawson 1959, S. 36). Aus dieser Komplementarität folge, dass nicht identifiziert werden kann, was keine raumzeitliche Platzierung hat. Solche „unbeobachtbaren“ (Strawson 1959, S. 34) nicht-dreidimensionalen Einzeldinge ließen sich nur vermittels einer Referenz auf die Einzeldinge einer anderen Klasse identifizieren. Als Paradigma für die Aufklärung dieser referentiellen Abhängigkeit benutzt Strawson den Begriff der Person. Der umfasse sowohl den Begriff des materiellen Körpers, das unseren Beobachtungs- und Lokalisierungsfähigkeiten entspricht, wie auch den Begriff der sog. „private particulars“ (unbeobachtbaren Einzeldinge, Strawson 1959, S. 41), mit denen Strawson alle mentalen Ereignisse und Wahrnehmungen meint und die sich als solche nicht ohne den Rekurs auf andere Typen von Einzeldingen lokalisieren ließen. Auf diese Weise seien „die Prinzipien der Individualisation“" (Strawson 1959, S. 41) von privaten Einzeldingen in Individualisierungsprinzipien der Personen fundiert, denen sie gehören. Jede erfolgreiche Referenz auf ein solches abhängiges Einzelding (z. B. Schuldgefühl oder Zahnschmerz) impliziere die Referenz auf eine lokalisierte Person. ${ }^{3}$

Wenn aber der Hinweis auf ein „mentales Ereignis“ (Strawson 1959, S. 41) in diesem Fall einen Hinweis auf die Person impliziert, die es „hat“, gilt nicht dasselbe für jede Referenz (insofern jede Referenz in der Ostension gründet)? Gilt die Äquivalenz: diese Erinnerung = die Erinnerung, die die Person A hat, gilt dann nicht dieselbe deskriptive Gleichheit: dieser Baum = der Baum, den jetzt die Person A sieht? So lebt sich nun Strawson in die Rolle eines potentiellen Opponenten ein, der die Auffassung vertritt, dass jede Ostension einen Wahrnehmenden impliziert (Strawson 1959, S. 42). Folgt

2 Humes Skeptizismus sei wie jeder „Revisionismus“ widersinnig, da er in der Formulierung des Einwandes gegen das gültige „Begriffsschema“ dieses Schema gültig macht. Wenn man mit Hume die numerische Identität der materiellen Dinge leugnet, schaffe man laut Strawson auch „the idea of a single spatio-temporal system of material things“ ab, das erst einen Zweifel an der numerischen Identität der Dinge möglich macht. (Vgl. Strawson 1959, S. 35: „Now I say that a condition of our having this conceptual scheme is the unquestioning acceptance of particular-identity in at least some cases of non-continuous observation. Let us suppose for a moment that we were never willing to ascribe particular- identity in such cases. Then we should, as it were, have the idea of a new, a different, spatial system for each new continuous stretch of observation. (Most of the common concepts of material things that we have would not exist; for the continuous stretches of observation that do occur are not long enough or comprehensive enough to allow of any use for them.) Each new system would be wholly independent of every other. There would be no question of doubt about the identity of an item in one system with an item in another. For such a doubt makes sense only if the two systems are not independent, if they are parts, in some way related, of a single system which includes them both. But the condition of having such a system is precisely the condition that there should be satisfiable and commonly satisfied criteria for the identity of at least some items in one sub-system with some items in the other.")

3 "The principles of individuation of [such private] experiences essentially turn on the identities of the persons to whose histories they belong. A twinge of toothache or a private impression of red cannot in general be identified in our common language except as the twinge which such-and-such an identified person suffered or is suffering, the impression which such-and-such an identified person had or is having. Identifying references to 'private particulars' depend on identifying references to particulars of another type altogether, namely persons" (Strawson 1959, S. 41). 
also aus diesen deskriptiven Äquivalenzen nicht, dass alle Typen von Einzeldingen prinzipiell abhängig sind, d. h. dass man sich auf sie nicht anders beziehen kann als durch die implizierte und fundierende Referenz auf eine radikal differente Einzeldingsart, nämlich auf ein wahrnehmendes (die Ostension durchführendes) Einzelding? Strawson leugnet natürlich nicht die Einbettung des Wahrnehmenden in jede Ostension, er hebt aber (im Sinn von Moore) hervor, dass diese Tatsache die identifizierbaren Einzeldinge nicht nivelliert und dass die Differenz abhängig-unabhängig durchzuhalten ist: referieren die Person A und die Person B in einem Gespräch auf „diesen Baum“ (Strawson 1959, S. 42), dann unterscheidet sich diese Identifikation von dem Fall, in dem diese Personen von einem Gedanken oder von den Zahnschmerzen der Person C sprechen. Die deskriptiven Wesen dieser beiden Referenzen sind insofern verschieden, dass der Unterschied zwischen abhängigen und unabhängigen Einzeldingen laut Strawson genügend motiviert ist.

Ebenso wie die privaten Einzeldinge seien auch alle theoretischen Konstruktionen (z. B. die Partikeln in der Physik) abhängig von der Identifikation von anderen wahrnehmbaren Körpern. Allgemeiner formuliert: Alle abstrakten oder komplizierten Einzeldinge hängen, was ihre Identifikation betrifft, von den weniger komplizierten ab. ${ }^{4}$ (Ein Beispiel aus den Sozialwissenschaften: Das Einzelding „Streik“ hänge identifikationsgemäß von „Menschen“, Fabriken“, „Maschinen“ usw. ab; vgl. (Strawson 1959, S. 44).

Strawson resümiert: Damit Elemente einer Einzeldingsart unabhängig identifizierbar sein können, müssen sie zwei Bedingungen erfüllen: Sie dürfen weder „privat“ noch „unbeobachtbar" ${ }^{\text {5 }}$ sein (Strawson 1959, S. 53).

Mögliche Kandidaten, die diese Bedingungen erfüllen, sind Strawson zufolge Ereignisse und Zustände einerseits und materielle Objekte andererseits. Die Ereignisse und Zustände werden von Strawson jedoch sofort ausgeschlossen, weil sie mit der Darbietung von einem typengemäß homogenen Rahmen ein Problem haben (sie sind verschiedener Art) und erst recht keine Einheitlichkeit eines solchen Rahmens aufweisen (Strawson 1959, S. 53). Die materiellen Körper erfüllen dagegen erfolgreich die Aufgabe von fundamentalen Einzeldingen, da sie solch einen Rahmen bieten. Dass man über diese verfügt, belege unsere Fähigkeit, zumindest einige seiner Elemente zu identifizieren.

Die Errungenschaft der ersten Betrachtung Strawsons in Einzeldinge ist also die Erkenntnis, dass materielle Körper die fundamentalen Einzeldinge in unserer konzeptuellen Struktur sind. Und die Person muss infolgedessen (auch) ein materieller Körper sein, solange sie identifizierbar ist.

Von dieser Sichtweise geht Strawson aus, wenn er das fundamentale Problem der Existenz bewusster Subjekte benennt; er drückt es aus in zwei Fragen:

1. Warum schreiben wir überhaupt einem Gegenstand mentale Charakteristiken zu?

4 „From this there follows immediately a general identifiability-dependence of particulars of the more sophisticated, upon particulars of the less sophisticated, type. For we could not speak of, and hence, identify, particulars of the more sophisticated type unless we could speak of, and hence identify, particulars of the less sophisticated type“ (Strawson 1959, S. 44f).

5 „The minimum conditions of independant identifiability for a type of particulars were that its members should be neither private nor unobservable" (Strawson 1959, S. 53). 
2. Warum schreiben wir sie demselben Ding zu, dem wir auch die physischen Charakteristiken zuschreiben (Strawson 1959, S. 90)?

Personen unterscheiden sich Strawson zufolge von anderen Objekten vor allem durch die sensomotorische Koordination, oder, besser ausgedrückt, durch die kinästhetischen Korrelationen. Also nicht nur dadurch, dass sie mit mentalen (es werden ihnen Absichten, Wahrnehmungen, Gedanken, Gefühle zugeschrieben) wie physischen Attributen ausgestattet sind, sondern in erster Linie dadurch, dass der Körper von Personen als Subjekt zweier Reihen kategorial differenter Prädikate zu einer sinnvollen Kommunikation fähig sei gerade zwischen der Sphäre des Bewusstseins (oder der Wahrnehmung) und der der materiellen Welt. Eben die kinästhetischen Korrelationen, wie sie z. B. durch Augenlider-, Augen- und Kopfbewegungen verwirklicht werden und wie diese in der Wahrnehmungsperspektive zum Ausdruck kommen, ließen die Körper von Personen zu den ausgezeichneten materiellen Einzeldingen werden (Strawson 1959, S. 90). Die kinästhetischen Korrelationen bedeuten, dass die sinnliche Erfahrung „[... von der Lokalisierung wie dem Zustand bestimmter Organe eines und desselben Körpers abhängen “. ${ }^{6}$ Es lasse sich somit sagen, dass „es für jede Person einen Körper gibt, der eine bestimmte Stellung der sinnlichen Erfahrung derselben Person gegenüber einnimmt“" ${ }^{7}$

Insofern sind, meint Strawson, die Gründe erfasst, warum man von allen Dingen gerade den einen für den eigenen Körper hält, also gerade den seinen; die fundamentale Frage, wie man überhaupt einen Begriff des Ich haben kann bzw. wie man überhaupt einem Individuum Erfahrungen oder mentale Zustände zuschreiben kann, werde damit aber noch nicht berührt (vgl. Strawson, 1959, S. 92). Wir wissen bisher vom Ich nur, dass ihm sowohl mentale als auch physische Eigenschaften zugeschrieben werden (und dass diese kinästhetisch zusammenhängen).

Strawson nennt nun ein Kriterium für jede Theorie der Identifikation des Subjekts (der Person). Dies sei die Fähigkeit zu erklären, dass wir uns die Zustände nur unter der Bedingung zuschreiben können, dass wir imstande sind, sie anderen Menschen zuzu-

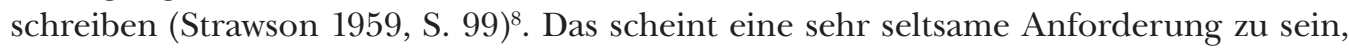
zumal sie sich nur auf "the dictionaries“ (Strawson 1959, S. 99) beruft, darauf, dass die Verwendung von psychischen Prädikaten von der semantischen Invarianz bedingt und

6 „But there is still the double dependence of the character of the experience on both the location and the state of certain organs of one and the same body" (Strawson 1959, S. 91).

7 „We may summarize such facts by saying that for each person there is one body which occupies a certain causal position in relation to that person's perceptual experience, a causal position which in various ways is unique in relation to each of the various kinds of perceptual experience he has; andas a further consequencethat this body is also unique for him as an object of the various kinds of perceptual experience which he has" (Strawson 1959, S. 92).

8 Vgl.: „it is a necessary condition of one's ascribing states of consciousness, experiences, to oneself, in the way one does, that one should also ascribe them, or be prepared to ascribe them, to others who are not oneself.“ (Strawson 1959, S. 99). Und Strawson setzt erklärend fort: „It means, for example, that the ascribing phrases are used in just the same sense when the subject is another as when the subject is oneself. Of course the thought that this is so gives no trouble to the nonphilosopher: the thought, for example, that 'in pain' means the same whether one says 'I am in pain' or 'He is in pain'. The dictionaries do not give two sets of meanings for every expression which describes a state of consciousness: a first-person meaning and a secondand-third person meaning" (Strawson 1959, S. 99). 
beherrscht wird. Das Verwenden des Prädikats „Zahnschmerzen haben“ sei nämlich gleich, unabhängig davon, ob man es in der ersten Person auf sich bezieht oder von einem anderen aussagt. Strawson fragt selbst, wie hier die Identität der Bedeutung erhalten bleiben kann, wenn die Verifikationsmethode so different ist. Im Falle der ersten Person fehle sie völlig (man weiß von seinem eigenen Zahnschmerz unmittelbar), im Fall der zweiten oder dritten gründe sie sich auf der Beobachtung eines Verhaltens. ${ }^{9}$

Strawson meint, dass die semantische Invariante damit zusammenhängt, dass „wir primär mit anderen, wegen der Informationen von den anderen sprechen "10, was die Tatsache erkläre, dass wir uns die Zustände darum zuzuschreiben imstande sind, weil wir sie den anderen zuschreiben können (Strawson 1959, S. 99).

Die recht unübersichtliche Betrachtung Strawsons lässt sich also folgendermaßen zusammenfassen: Die Zustände können wir uns zuschreiben, weil wir sie anderen zuschreiben können. Anderen können wir sie nur dann zuschreiben, wenn wir sie als Subjekte der Erfahrung identifizieren. Wir identifizieren sie jedoch nicht, wenn wir sie nur als Subjekte der Erfahrung (als Eigentümer des Bewusstseins) identifizieren wollen (Strawson 1959, S. 100) ${ }^{11}$. Darin wird ein Angriff auf Descartes realisiert (Strawson 1959, S. 100) und eine Lösung gefunden, da es hierbei im Unterschied zu dem Cartesianismus und ebenfalls der sog. subjektlosen Theorie gelinge, die Einmaligkeit der psychischen Zustände aufzuklären. Die Lösung besteht in der Annahme der Existenz einer solchen Entität, der man Bewusstseinszustände wie Körpercharakteristiken zuschreiben kann, und in der angeblichen Primitivität ihres Begriffs. Die Antwort auf die oben gestellten Ausgangsfragen bekommt auf diese Weise die folgende Gestalt: „Bewusstseinszustände lassen sich nur solchen Dingen zuschreiben, denen bestimmte Körpereigenschaften zugeschrieben werden“ (Strawson 1959, S. 102)12, also nun den „Personen“ (Strawson 1959, S. 102). Damit werde die Eigenartigkeit des Begriffs eines rein individuellen Bewusstseins ausgeschlossen, das höchstens ein Teil des primären Personbegriffes sein könne (da es nicht individualisierbar sei, d. h. man könne ihm keine Erfahrungen zuschreiben). Und somit sei bewiesen, dass „ich“ nur auf eine „Person“ referiert. ${ }^{13}$

In dieser Betrachtung bleibt rätselhaft, wie das, was ich in meinem Fall unmittelbar weiß, „bedingt“ werden kann durch die Fähigkeit, es anderen zuzuschreiben. Wie kann ein unmittelbares Bewusstsein in der Beobachtung eines anderen fundiert werden? ${ }^{14}$

9 „How could the sense be the same when the method of verification was so different in the two cases -or, rather, when there was a method of verification in the one case (the case of others) and not, properly speaking, in the other case (the case of oneself)? (Strawson 1959, S. 100)

$10,[\ldots]$ we speak primarily to others, for the information of others" (Strawson 1959, S. 100).

11 Vgl.: „One can ascribe states of consciousness to oneself only if one can ascribe them to others. One can ascribe them to others only if one can identify other subjects of experience. And one cannot identify others if one can identify them only as subjects of experience, possessors of states of consciousness" (Strawson 1959, S. 100).

$12,[\ldots]$ a necessary condition of states of consciousness being ascribed at all is that they should be ascribed to the very same things as certain corporeal characteristic" (Strawson 1959, S. 102)

13 Der Ausdruck ist also nicht homonym.

14 Strawson kann hier nur die logische Relation der gegenseitigen Implikation zweier Fähigkeiten im Sinne haben (die von der semantischen Identität der Prädikate abgeleitet wird, die in verschiedenen epistemischen Positionen erhalten sei). 
Auch Strawsons Schüler Ernst Tugendhat geht es darum, die psycho-logische SelbstZugänglichkeit in das raumzeitliche Netz zu übertragen und das Problem des Selbstbewusstseins (bzw. der epistemisch transparenten, d. h. selbstbewussten, Erlebnisse) durch eine terminale grammatische Reinstallation verständlich zu machen: Die raumzeitliche Identiät des Ich wird durch das System der deiktischen Pronomina gesichert. Das Ich nimmt sich nicht wahr, hängt jedoch systematisch mit $D u$ zusammen, das wahrgenommen wird, die Identität des Ich ist somit Identität des wahrnehmbaren Ich als Identität des wahrgenommenen $D u$ bzw. Er (aus der Sichtweise des Anderen), wodurch sich das ich in einen identifikationellen Rahmen einlegt, der die Intersubjektivität (d. h. für Strawson und Tugendhat: Identität) stifte (Tugendhat 1979, S. 70).

Das Problem der epistemischen Asymmetrie lösen die sprachanalytischen Denker also durch die Annahme einer semantischen veritativen Symmetrie. Das grammatische Apriori des Systems der deiktischen oder persönlichen Pronomina (darin bestehend, dass sich ein mit einem deiktischen Ausdruck bezeichneter Gegenstand grundsätzlich auch mit einem anderen deiktischen Ausdruck derselben Art bezeichnen lässt) sei also die Gewähr der Intersubjektivität und der Identität des Subjekts.

\section{Bedeutungswandel durch die epistemische Asymmetrie (Frank)}

Die hier von den analytischen Denkern angestrebte Erklärung der epistemischen Asymmetrie unterzog Tugendhats (und Gadamers) Schüler Manfred Frank einer triftigen Kritik aus der hermeneutischen Position. Es müsste laut Frank zuerst gezeigt werden, dass Ich und $\mathrm{Du}$, die beide dasselbe Individuum durch ein eindeutiges Einreihen in das raumzeitliche Netzwerk identifizieren, nicht Verschiedenes bezeichnen können. Nach Frank hat die formal-semantische Symmetrie in der Situation von radikal differenten Verifikationsweisen nicht den von Strawson und Tugendhat suggerierten veritativen Charakter, sondern sie bedeute nichts mehr als einen willkürlichen „Konditional“: „Es ist klar, dass, wenn ich auf die semantische Identität zwischen den $\psi$-Prädikaten hinauswill, ich den verschiedenen epistemischen Standpunkten, aus denen diese Prädikate zugesprochen werden, kein sehr hohes Gewicht zumessen kann“ (Frank 1986, S. 87). Die semantische Symmetrie sei eine bloße Annahme und keineswegs epistemisch ausgewiesen.

Für Frank ist die Voraussetzung einer veritativ-adäquaten Intersubjektivität sowie die Bagatellisierung der epistemischen Asymmetrie ein Beleg des naiven Beharrens auf einem vor-hermeneutischen (vor-schleiermacherschen) Kommunikationsmodell, das annimmt, dass Verständigen über die Welt und über sich die „Identität grammatischer Regeln und sprachlich schematisierter Vorstellungen" fordert, was aber in der Tat die Kommunikation unmöglich machen und sie in einen ahistorischen „Monolog der Grammatik" verwandeln würde (Frank 1986, S. 89). Die Hermeneutik werde demgegenüber dem Umstand gerecht, dass menschliche Verständigung ein Prozess von stetigen Sinntransformationen und neuen Sinnschematisierungen ist, dass immer neue Bedeutungsverschiebungen und Erkenntnisfortschritte stattfinden und dass spezifische Sichtweisen 
gebildet werden, die in Strawsons und Tugendhats Welt der erstarrten Semantik keinen Platz hätten (vgl. Frank 1986, S. 87).

Aus der auch von Strawson und Tugendhat anerkannten Absenz der Identifikation innerhalb des Selbstbewusstseins folge bereits die Unhaltbarkeit der ahistorischen semantischen Identität, nämlich dass ein selbstbewusstes Erlebnis durch keine auf der Identifikation gegründete Beschreibung des Körperzustandes (geschweige denn aus der Perspektive eines anderen) zu erfassen ist und dass es somit die suggerierte Konvertibilität beider Perspektiven nicht gibt, meint Frank. Zwischen diesen Perspektiven bestehe eine einseitige Abhängigkeit: Wenn ich imstande bin, mich mithilfe der Körpereigenschaft (im Prinzip mit einem Irrtumsrisiko) zu identifizieren ${ }^{15}$, bin ich schon imstande (gewesen), mir ohne Identifikation (irrtumslos) Erlebnisse oder psychische Eigenschaften ${ }^{16} \mathrm{zu}-$ zuschreiben. Der sog. Subjektsgebrauch der Prädikate (die selbstbewusste Anwendung psychischer Eigenschaften) gehe dem Objektgebrauch (Anwendung der Prädikate, mit denen ich mich als Gegenstand identifiziere) voraus und ermögliche ihn.

Da sich ein Subjekt seine Eigenschaft nicht aufgrund der (Selbst-)Beobachtung zuschreibt, sondern dies (nach Strawson, Tugendhat sowie Frank) unmittelbar erfolgt (d. h. es ist sich dessen direkt bewusst), entstand die Frage danach, wie ein unmittelbar Gewusstes mit dem Beobachteten (z.B. aus der Position der dritten Person) zu identifizieren ist (z. B. unter dem Namen „Zahnschmerzen“). Frank lehnt es ab, dass die Identität des in den epistemisch differenten Perspektiven Gegebenen durch die Semantik der Wörter oder systemfesten Regeln (z.B. des Gebrauchs der Pronomina) gewährleistet ist. Strawsons Annahme, das von der ersten Person her unmittelbar Gewusste sei von dem in der dritten Person Beobachtete abhängig (das Unmittelbare sei vom Vermittelten bedingt, also das Gewisse vom Hypothetischen), muss Frank natürlich für offensichtlich falsch halten. Ein mentales Ereignis lässt sich keineswegs über materielles identifizieren, da die Identifikation des letzteren epistemisch minderwertig ist. Strawson scheint das nicht zu sehen, und zwar aufgrund seines Materialismus bzw. des strukturalistisch intersubjektivistischen Dogmas, das bis zu Hegel führt (Selbstbewusstseine entstünden miteinander und durcheinander, bzw. Identität komme negativ-diferentiell zustande). Die Nivellierung der so verschiedenen epistemischen Perspektiven ist eine Folge dieser metaphysischen Belastung. Eine Bedingung der raumzeitlichen Selbst-Lokalisierung ist also die subjektive Bekanntschaft des Bewusstseins mit sich selbst, Strawson-Tugendhats raumzeitliches Netz kann man folglich als Auslegungsrahmen der Individualisierung nicht gebrauchen, lesen wir in Franks Argumentation.

Weil die nicht-identifizierende Bekanntschaft unabhängig und original ist (infolgedessen können Aussagen über Bewusstsein durch Aussagen über beobachtbares Verhalten nicht vertreten werden), lässt sich die Subjektivität auf raumzeitliche Identität nicht reduzieren. Und weil die „erste Person“ die Voraussetzung der zweiten und der dritten ist und weil sich niemand aus der eigenen Perspektive zu identifizieren braucht (und aus der eines anderen nicht identifizierbar ist), ist es Frank zufolge nicht möglich, dass 
sich Personen durch Identifikation individualisieren, und „die Identität [ist] überhaupt kein Definiens von Individualität” (Frank 1986, S. 98), wodurch das analytische Projekt, gegründet auf semantischer Identität der die Individuen-Personen identifizierenden Prädikatsbedeutungen, haltlos wird.

Strawsons und Tugendhats grammatischer Begriff der fixen Identität soll natürlich nicht nur die Person erfassen, sondern auch die Semantik der ihr zugeschriebenen Prädikate, deren inter-perspektive Identität erst die intersubjektive Identifikation der Person gewährleistete. Diese scheint in ihrer Auffassung ein (verschieden kombiniertes) Depot von Elementen invarianter Semantik zu sein, was für den Hermeneutiker unakzeptabel ist. ${ }^{17}$

Frank tritt also scharf gegen Strawsons semantischen Sensualismus auf: Sinnliche Wahrnehmung bietet keine Bedeutungen, geschweige denn die für alle identischen, sondern Bedeutungen sind ein Ergebnis der hermeneutischen Anstrengung, die nicht versöhnbar ist mit einer problemlosen Postulierung der Identität der Beobachtungs(verifikationellen) Positionen, weil auch dieselbe Stellung in Strawsons raumzeitlichem Sinn natürlich der Unendlichkeit der Auslegungen dessen ihren Raum lässt, was darin "gegeben“ ist, solange dieser Ausdruck für Hermeneutiker noch zu halten ist.

Bedeutungen „fallen“ also „nicht mit einer fixen semantischen Identität versehen aus dem Ideenhimmel“, sondern „sie werden Ausdrücken durch Sinn-Hypothesen in prinzipiell widerrufbarer Weise verliehen“ (Frank 1986, S. 101). Die Bedeutung ist eine hypothetische Auslegung.

„Meine an den anderen oder die von ihm an mich gerichtete Stimme ist doch nur unter der Bedingung als die eines anderen oder an einen anderen erkennbar, dass sie aus meiner (oder seiner) alleinigen Regelbeherrschung nicht hätte erzeugt werden können. Das Code-Modell der Sprache, das die bedeutungsindifferente Übersetzbarkeit aller Aussagen aus der 'er'- in die 'ich'-Perspektive und umgekehrt unterstellt und die semantische Innovation durch den Typenbegriff überwacht (d. h. sanktioniert) ist so nur scheinbar intersubjektiv engagiert. Dies Modell macht die Anderheit des Anderen fiktiv: Er mag sagen, was er will: sofern seine Äußerung in nichts Anderem besteht als in der Übermittlung einer Information oder in der Durchgabe eines konventionalisierten Handlungsschemas, ('Ich habe Schmerzen', 'Ich mag dich', 'Ich denke, dass...', 'Ich verspreche dir, dass...', 'Gib dir Mühe'), sofern sie vielmehr eine individuelle Deutung von Sachverhalten mitzuteilen unternimmt, hat der Code sie semantisch-pragmatisch immer schon vorgesehen. Ich hätte sie, unter geeigneten Umständen, ebenso formen können. Die Ersetzbarkeit unserer Sprecher-Perspektiven (wie sie die technische Lehre von Sprecher-Hörer ratifiziert) nivelliert die Innovativität und die Sinnschöpfungsenergien jedes Dialogs und reduziert ihn aufs Aus-sagen des im gemeinsamen Repertoire Vorgesehenen. Weltdeutungs-, also hermeneutische Probleme sind von diesem linguistischen Paradigma schlicht nicht vorgesehen. Tugendhats Formulierung, wonach die epistemische Asymmetrie niemals semantische Auswirkungen haben könnte (dass unsere Äußerungen 'eine verschie-

17 Deshalb sucht Frank vom allgemeinen Dedukt der Person zu dem romantischen Begriff der „Individualität“ zu übergehen, der die „Zweifelhaftigkeit“ der ahistorischen Äquivalenz zeige (die von Strawson lapidar formuliert wird: gefühlt/unbeobachtet = ungefühlt/beobachtet (vgl. Strawson 1992, S. 124). 
dene Bedeutung haben, ist aber ausgeschlossen durch die veritative Symmetrie dieser Sätze' (Tugendhat 1979, S. 89)), zeigt die Verhärtung eines Postulats zu einem konstitutiven Prinzip. Sie ist das Resultat einer METABASIS EIS ALLO GENOS, keine notwendige Konsequenz aus einer auf Intersubjektivität blickenden semantischen Besinnung“ (Frank 1986, S. 121).

Die Idee der Auslegungsinitiative als letzter unüberschreitbarer Instanz, von der aus auch das Phänomen der Sprache verständlich gemacht wird, bringt Frank mit Hilfe des Gedankens der Apräsenz (der Verspätung) der Interpretation bis zu einem universellen semiologischen System. Die individuelle Invention des Interpreten bewirke, was Frank auch bei seiner Kritik an Derridas Zeichenkonzeption zur Geltung bringt (vgl. Rubáš 2017, S. 79), dass das Zeichen selbst nicht-präsent ist („die Struktur oder das Zeichen kann nie mit sich selbst zusammenfallen“), weil die die Zeitlichkeit gleichsam in das Innere des Zeichens eintrage.

Franks zeitliche, mit der Notwendigkeit der freien selbstbewussten Individualität korrelierende Semiologie basiert anschaulich auf der Vorstellung einer dichotomen Zeichenstruktur (Zeichen - Bedeutung), die aber eine nicht ganz überprüfte Idee suggeriert, nämlich dass das Zeichen Gegenstand der Interpretation sei.

Von den Zeichen, die bei Interpretation in der erklärenden oder hypothetisch-erklärenden Funktion verwendet werden, kann man keinesfalls sagen, sie verfallen gleichermaßen dem Zeitabstand von deren Bedeutungen wie ein Zeichen-Gegenstand. Das Sprachzeichen nimmt nämlich vor allem an der die Wirklichkeit fassenden und auslegenden Tätigkeit teil, ein Rebus ist es primär nicht. Das Zeichen, mit dem ich eine Bedeutung ausdrücke, interpretiere ich nicht, es ist für mich kein transzendentes Rätsel, dessen Bedeutung ich erst suche. Den Dialog als Vorbild des Sprachgebrauchs zu wählen und eine Semiologie darauf zu bauen, ist möglich (und willkürlich), aber selbst Frank zeigte durch die Bevorzugung des Subjektgebrauchs vor dem Objektgebrauch, dass die „Interpretation“ nicht die Basis der sprachlichen Handlung ist: Wenn ich meinen mentalen Zustand ausdrücke, drücke ich ihn sprachlich aus, ich spreche, allerdings interpretiere ich nichts, ich identifiziere nichts, ich errate nicht, ich lege nicht aus und erstelle keine Hypothesen. Somit legt Franks Beweisführung selbst Schleiermachers Konzeption der Beziehung zwischen Subjekt und hermeneutischer Sprache als unakzeptabel offen.

\section{Zeichenlehre aufgrund der epistemischen Asymmetrie (Husserl)}

Frank wies darauf hin, dass Strawsons Annahme der semantischen Gleichheit der Prädikate, die aus den verschiedenen epistemischen Positionen (der Subjekt- und der Objektperspektive) zugeschrieben werden, mit einer anderen Voraussetzung Strawsons kollidiert, und zwar mit der der direkten Zugänglichkeit der Inhalte aus der Subjektperspektive (die nach Strawson sogar durch die interpretatorische Objektposition berichtigt sein sollte). Wir haben aber gezeigt, dass Franks Annahme der Priorität und Irreduzibilität der epistemischen Position der ersten Person eine andere Sprach- oder Zeichentheorie verlangt als diejenige, die das Zeichen als (je in die Vergangenheit sinkendes) 
Objekt der Interpretation fasst. Sie verlangt sogar die gegensätzliche Zeichenkonzeption. Franks hermeneutische Zeichenauffassung ist somit im Widerspruch mit seiner These der Vorrangstellung der Subjektposition (die die Inhalte interpretationslos erfasst). Da Edmund Husserl in seinen früheren Texten seine Zeichentheorie im ausdrücklichen Zusammenhang mit den Untersuchungen der Sphäre der unmittelbaren (interpretationslosen) Selbstgegebenheit der Gehalte in der Perspektive der ersten Person formuliert hat, reihen wir hier eine kurze Erinnerung an diesen subtilen Denker ein. Wie fasst Husserl, der die epistemische Asymmetrie wirklich ernst nimmt, das Zeichen auf?

Husserl geht vor allem davon aus, dass eine wissenschaftliche Theorie sich nicht in den Köpfen der Wissenschaftler in verschiedene subjektive Versionen zerfällt. Sie bleibt objektiv dieselbe, unabhängig davon, wer sie denkt: logische (semantische) Entitäten werden in einzelnen Denkakten nicht vermehrt, sie werden in ihnen als identische behalten.

Auf der anderen Seite ist für Husserl der Begriff des psychischen Phänomens grundlegend, das wesentlich durch Intentionalität gekennzeichnet ist. Die Intentionalität ist die Eigenschaft des psychischen Phänomens, auf einen Gegenstand gerichtet zu sein. Husserls Grundproblem besteht in der Beziehung zwischen den objektiven Bedeutungen (die z. B. als wissenschaftliche Begriffe oder Wahrheiten auftreten) und den psychischen Phänomenen des Denkens oder Erkennens. Die letzteren sind subjektiv und haben einen prozessualen Charakter (des Aktes). Daraus resultieren Husserls Fragen, die das Hauptthema seiner Logischen Untersuchungen (Husserl 1993) sind: Wie wird das Objektive in den subjektiven Vollzügen (des Meinens, Vorstellens, Erinnerns usw.) gegeben oder „konstituiert“? Wie kann man überhaupt die Sätze der Wissenschaft oder Logik denken (vgl. z. B. Husserl 1993a, S. VII)?

Husserls Lösung des Problems der Beziehung zwischen dem subjektiven psychischen Erlebnis und der objektiven Bedeutung besteht darin, dass das letztere seine einzelne Instanz im ersteren findet. Die logische Bedeutung ist „Bedeutungsspezies des objektivierenden psychischen Aktes“. Das Verhältnis ist dasselbe wie zwischen dem konkreten, einzelnen Vorkommen des Roten und dem Rot als solchem (Husserl 1993b, S. 106). Das eine Objektive erscheint also mehrfach in psychischen Erlebnissen wie Platons Idee in der Welt der Veränderung und der sinnlichen Wahrnehmung.

Weil logische Strukturen und Kategorien in Verbindung mit sprachlichen Zeichen gegeben werden, befasst sich Husserl bei seinen Erörterungen auch mit der Sprache. Dabei legt er einige wichtige semiologische Unterscheidungen vor, vor allem die Differenzierung von Anzeichen und Ausdruck, die für uns hier am interessantesten ist:

\footnotetext{
„Alle Anzeichen haben gemeinsam den Umstand, dass irgendwelche Gegenstände oder Sachverhalte, von deren Bestand jemand aktuelle Kenntnis hat, ihm den Bestand gewisser anderer Gegenstände oder Sachverhalte in dem Sinne anzeigen, dass die Überzeugung von dem Sein der einen von ihm als Motiv (und zwar als ein nichteinsichtiges Motiv) erlebt wird für die Überzeugung oder Vermutung vom Sein der Anderen“ (Husserl 1993, S. 25).
}

Diese Rede über Motivation innerhalb des semiotischen Prozesses stellt Husserls Ausgangspunkt gleich in die Nähe von Franks abduktionistischem Subjektivismus (und 
distanziert ihn von Derridas Naturalismus, der sich auf die Verallgemeinerung der „anzeichnenden“, hinweisenden Funktion der sprachlichen Ausdrücke gründet). Die Allgegenwärtigkeit der kundgebenden Funktion, wie sie Husserl nennt, bestreitet er nicht, ohne mit ihr die eigentliche Leistung der Ausdrücke gleichzustellen:

„Wenn man diesen Zusammenhang überschaut, erkennt man sofort, dass alle Ausdrücke in der kommunikativen Rede als Anzeichen fungieren. Sie dienen dem Hörenden als Zeichen für die ,Gedanken“ des Redenden, d. h. für die sinngebenden psychischen Erlebnissen desselben, sowie für die sonstigen psychischen Erlebnisse, welche zur mitteilenden Intention gehören. Diese Funktion der sprachlichen Ausdrücke nennen wir die kundgebende Funktion“ (Husserl 1993, S. 33).

Die kommunikative Funktion der Ausdrücke beruht darauf, dass sie als Anzeichen wirken. „Aber auch in dem sich im Verkehr nicht mitteilenden Seelenleben ist den Ausdrücken eine große Rolle beschieden“ (Husserl 1993, S. 35):

\begin{abstract}
„Hiernach scheint es klar, dass die Bedeutung des Ausdruckes, und was ihm sonst noch so wesentlich zugehört, nicht mit seiner kundgebenden Leistung zusammenfallen kann. Oder sollen wir etwa sagen, dass wir auch im einsamen Seelenleben mit dem Ausdruck etwas kundgeben, nur dass wir es nicht einem Zweiten gegenüber tun? Sollen wir sagen, der einsam Sprechende spreche zu sich selbst, es dienten auch ihm die Worte als Zeichen, nämlich als Anzeichen seiner eigenen psychischen Erlebnisse?“ (Husserl 1993, S. 35-36).
\end{abstract}

Nach Husserl ist diese Sicht zurückzuweisen, denn das „Dasein des Zeichens motiviert nicht [...] unsere Überzeugung vom Dasein der Bedeutung“ (Husserl 1993, S. 36).

Es wäre natürlich absurd, zu meinen, dass man das Aussprechen eigener Worte abwarten muss, damit man erfährt, was man sagt: Die von mir artikulierten Ausdrücke sind für mich keine Gegenstände der Interpretation, wie dies bisher bei Frank der Fall zu sein schien. Auch im einsamen Seelenleben werden sicher Zeichen (als Ausdrücke) gebraucht. Selbst wenn wir annehmen, dass dieser Gebrauch notwendig ist, folgt daraus nicht, dass die Zeichen auf Anzeichen (marques) zurückzuführen sind bzw. dass sie als Gegenstände der Interpretation verständlich gemacht werden können. Husserl geht allerdings davon aus, dass die Ausdrücke im einsamen Monolog nicht notwendig sind:

„Die Nicht-Existenz des Wortes stört uns nicht. Denn zur Funktion des Ausdrucks als Ausdruck kommt es darauf gar nicht an. Wo es aber darauf ankommt, da verbindet sich mit der bedeutenden eben auch die kundgebende Funktion: der Gedanke soll nicht bloß in der Weise einer Bedeutung ausgedrückt, sondern auch mittels der Kundgabe mitgeteilt werden; was freilich nur möglich ist im wirklichen Sprechen und Hören. [...] In der monologischen Rede können uns die Worte doch nicht in der Funktion von Anzeichen für das Dasein psychischer Akte dienen, da solche Anzeige hier ganz zwecklos wäre. Die fraglichen Akte sind ja im selben Augenblick von uns selbst erlebt" (Husserl 1993, S. 36-37). 
Wir können somit gegen Frank (und gegen Derrida) ${ }^{18}$ konstatieren, dass die image acoustique im Monolog Ausdruck, kein Anzeichen (marque) ist.

Daraus ist zu sehen, dass die Originalität oder Unabhängigkeit der epistemischen Subjektposition mit sich bringt zugleich die Unaufgebbarkeit der Husserlschen Immanenz und Adäquatheit (als Sphäre der direkt oder unmittelbar gegebenen Inhalte, Franks und Strawsons Subjektposition), und Husserls Ausdruck-Begriff gehört organisch dazu (weil wenn die erste Person eigene/apodiktisch gegebene Gehalte mitteilt / sie ausspricht, benutzt sie Zeichen (im Monolog), ohne sie zu interpretieren, also als transparente Zeichen). Das bedeutet aber, dass das Zeichen nicht allgemein für ein zu Interpretierendes gehalten werden kann, und es entsteht darüber hinaus die rhetorische Frage, ob der unvermeidliche Zeichenbegriff Husserls mit dem alle Identität stets verwischenden, alles anarchisierenden Prinzip der Individualität und des Abduktionismus nicht kollidiert.

\section{Schluss}

Alle drei von uns erörterten Sprachtheoretiker (Strawson, Frank, Husserl), die sehr differenten philosophischen Strömungen angehören, sind sich der unterschiedlichen epistemischen Voraussetzungen des Subjekt- und des Objektgebrauchs der Prädikate bewusst. Sie sind sich auch darin einig, dass bei der Selbstzuschreibung der Subjektgebrauch der Prädikate dem Objektgebrauch vorausgeht und ihn bedingt. Diesen erkenntnistheoretischen Befund bringen sie aber in je ganz anderen Zusammenhang mit der Tatsache der semantischen (In)Stabilität der Bedeutungen der sprachlichen Einheiten in deren Gebrauch. Im sprachanalytischen Ansatz Strawsons wird die semantische Invarianz der Prädikate zweckdienlich vorausgesetzt, um die epistemische Asymmetrie unschädlich zu machen hinsichtlich ihrer unheilvollen Konsequenzen für den Kommunikationsbegriff als Austausch intakter Bedeutungen. Die hier wiedergegebene Kritik des Hermeneutikers Frank an Strawson hat die Dissonanz zwischen Strawsons Annahme zweier ganz verschiedener Verifikationsmethoden und Strawsons Idee der in der Kommunikation vorkommenden invarianten Bedeutungen gesehen. Franks alternative Auswertung des behandelten epistemischen Phänomens (als Begründung des Bedeutungswandels bzw. der Instabilität der Bedeutungseinheiten), haben wir als widersprüchlich offengelegt, weil der epistemische Vorrang der nicht-interpretierenden Subjektperspektive unverträglich ist mit der allgemein angelegten semiologischen Annahme Franks, die sprachlichen Zeichen seien Gegenstände der Interpretation. Husserls „Ausdrucks“-Theorie halten wir demgegenüber für eine mit der Vorrangstellung der Subjektperspektive kompatible Semiologie.

18 Bezeichnen (Ausdrücken) bedeutet bei Husserl also nicht Substituieren und es bedeutet somit auch nicht irgendeinen „supplément d'origine“ zu liefern, wie Derrida bei seiner Kritik an Husserl meint (Derrida 1993, S. 98). 


\section{Literaturverzeichnis:}

Derrida, Jacques (1993): La Voix et le Phénomène, Paris, PUF, Quadrige.

Frank, Manfred (1986): Die Unhintergehbarkeit von Individualität, Frankfurt am Main: Suhrkamp Verlag.

Frank, Manfred (1984): Was ist Neostrukturalismus?, Frankfurt am Main, Suhrkamp Verlag.

Frank, Manfred (1991): Selbstbewusstseinstheorien. Frankfurt, Suhrkamp Verlag.

Husserl, Edmund (1993a): Logische Unersuchungen. Prolegomena zur reinen Logik, Tübingen, Max Niemeyer Verlag.

Husserl, Edmund (1993b): Logische Untersuchungen, II/1, Tübingen, Max Niemeyer Verlag.

Rubáš, Michal (2017): Derrida und Saussure aus der hermeneutischen Perspektive als Ausgangspunkt für die Schlichtung eines diskurssemantischen Streites, in: Brünner Beiträge zur Germanistik und Nordistik 31 / 2017 / 2, Brno, Masarykova Univerzita.

Strawson, Peter Frederick (1974): Subject and Predicate in Logic and Grammar, London, Methuen.

Strawson, Peter Frederick (1959): Individuals, An Essay in Descriptive Metaphysics, New York, Routledge.

Strawson, Peter Frederick (1992): Analysis and Metaphysics, Oxford, Oxford University Press.

Tugendhat, E. (1979): Selbstbewußtsein und Selbstbestimmung, Frankfurt am Main, Suhrkamp Verlag.

Mgr. Michal Rubáš, Ph.D. / rubas@utb.cz

Univerzita Tomáše Bati, Fakulta humanitních studií, Ústav moderních jazyků a literatur

Štefánikova 5670, 76001 Zlín, Česká republika

This work can be used in accordance with the Creative Commons BY-SA 4.0 International license terms and conditions (https://creativecommons.org/licenses/by-sa/4.0/legalcode). This does not apply to works or elements (such as image or photographs) that are used in the work under a contractual license or exception or limitation to relevant rights 
